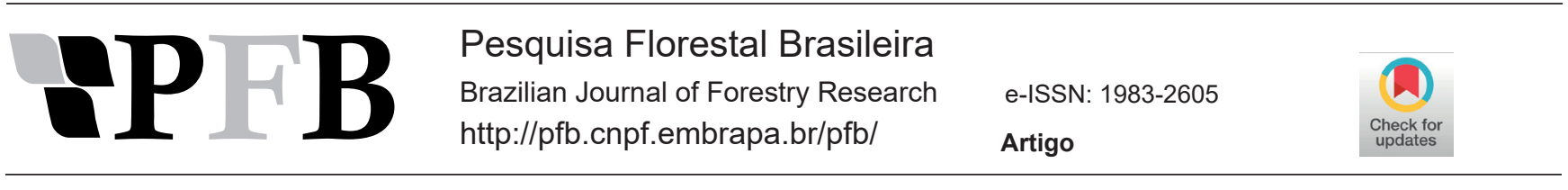

\title{
Deposição, decomposição e conteúdo de nutrientes de serapilheira em área de integração lavoura-pecuária-floresta na região do Cerrado
}

\author{
Fabiana Piontekowski Ribeiro ${ }^{1,2 *}$ (D), Karina Pulrolnik ${ }^{(D)}$, Lourival Vilela² ${ }^{(D)}$, Alcides Gatto ${ }^{1}$ (D) \\ ${ }^{1}$ Universidade de Brasília, Departamento de Engenharia Florestal, Campus Darcy Ribeiro, Asa Norte, CEP 70910-900, Brasília, DF, Brasil \\ ${ }^{2}$ Embrapa Cerrados, Rodovia BR 020, Km 18, CEP 73310-970, Brasília, DF, Brasil
}

"Autor correspondente:
fbn2.ribeiro@gmail.com

Termos para indexação:

Sistemas agroflorestais

Macronutrientes

Liteira

Index terms:

Agroforestry systems

Macronutrients

Litter

Histórico do artigo:

Recebido em 09/12/2019

Aprovado em 12/08/2021

Publicado em 25/02/2022
Resumo - Este trabalho teve por objetivo analisar a deposição, decomposição e os teores de nutrientes da serapilheira em área de integração lavoura-pecuária-floresta (ILPF), na Embrapa Cerrados, Planaltina, DF. Foram instalados coletores de serapilheira, e foram utilizados litterbags para avaliação da decomposição. Os coletores e os litterbags foram alocados entre árvores e entre renques. Em cada litterbag foram adicionados $20 \mathrm{~g}$ de serapilheira de eucalipto. As amostras de serapilheira de deposição e da decomposição foram secas e encaminhadas para laboratório, para análise de macronutrientes. A produção total de serapilheira foi de $9.394,2 \mathrm{~kg} \mathrm{ha}^{-1}$ entre árvores e $3.726,4 \mathrm{~kg} \mathrm{ha}^{-1}$ entre renques. A precipitação pluvial influenciou marcadamente a decomposição da serapilheira foliar. As taxas de decomposição foram similares para ambas posições, e o tempo de meia vida da decomposição em curto período e recalcitrante foram, respectivamente, de 10 e 1.386 dias entre árvores e de 10 e 1.155 dias entre renques. Os conteúdos de nutrientes ( $\mathrm{N}, \mathrm{P}, \mathrm{K}, \mathrm{S}, \mathrm{Ca}$ e $\mathrm{Mg}$ ) da serapilheira foram superiores na posição entre árvores. Desses, o $\mathrm{Ca}$ e o $\mathrm{N}$ foram os que apresentaram maiores conteúdos no processo de deposição e decomposição, independentemente da posição.

\section{Litter deposition, decomposition and nutrient content in a crop-livestock-forest integration area, in the Cerrado Region}

\begin{abstract}
This work aimed to analyze the decomposition and nutrient content of the litterfall in a crop-livestock-forestry integrated system (CLFI) located in Embrapa Cerrados, Planaltina, Distrito Federal, Brazil. Litter traps were distributed and litterbags were used to investigate the decomposition. The litter traps and litterbags were allocated between the tress and ranks. Each litterbag was filled with $20 \mathrm{~g}$ of eucalyptus litterfall. The samples of both collections were sent to a laboratory to determine the macronutrient content. The total litterfall was 9,394.2 $\mathrm{kg} \mathrm{ha}^{-1}$ between the tress and 3,726.4 kg ha-1 between ranks. The precipitation showed a high influence on the litterfall decomposition. The decomposition rates were similar to both positions and the half-life time in the short and long period (recalcitrant) were respectively 10 and 1,386 days between tress and 10 and 1,155 between ranks. The litterfall nutrient content (N, P, K, S, Ca and Mg) was high between trees. $\mathrm{Ca}$ and $\mathrm{N}$ showed greater content during the deposition and decomposition process independent of the position in the CLFI.
\end{abstract}




\section{Introdução}

O total de florestas plantadas no mundo soma 264 milhões de ha e vários são os objetivos dessas plantações, sendo $76 \%$ desses plantios destinados à produção madeireira (FAO, 2015). No Brasil, as florestas plantadas totalizavam 7,83 milhões de ha em 2018, sendo 5,7 milhões de ha com plantio de eucalipto. Essa espécie é importante nos programas de reflorestamento, devido ao seu rápido desenvolvimento (IBÁ, 2019), diferentes opções de manejo dos componentes orgânicos da colheita (Versini et al., 2013), ampla variedade de espécies e sua adaptabilidade a diferentes condições edafoclimáticas (Santos et al., 2013; IBÁ, 2019).

Os povoamentos de eucalipto são a principal fonte de produção de madeira no país (Gonçalves et al., 2013; Colodette et al., 2014), sendo uma das melhores matérias-primas para a produção de bioenergia (Ouyang et al., 2016). A alta produtividade desse monocultivo foi obtida por meio de extensa pesquisa nas últimas décadas, principalmente em melhorias de genética e silvicultura. Embora os plantios de eucalipto apresentem alta produtividade, é importante observar a elevada exportação de nutrientes durante a colheita (Versini et al., 2013; Gatto et al., 2014; Ferreira et al., 2016), o que pode comprometer a produtividade de ciclos futuros. Manter a fertilidade do solo em níveis adequados para cultivo tem um elevado custo financeiro e demanda conhecimento técnico sobre a necessidade nutricional de cada espécie a ser implantada (Martins et al., 2015).

Com o crescimento populacional e o aumento da demanda por alimentos, o aprimoramento de tecnologias na produção agrícola nos últimos anos é baseada apenas em sistemas simples de monocultura. Além disso, o aumento do manejo mecanizado de forma intensiva tem mostrado sinais de saturação do solo (Balbino et al., 2011a). Para diminuir a demanda por recursos naturais, o sistema de integração lavoura-pecuária-floresta (ILPF) torna-se um instrumento à disposição do agronegócio e de interesses nacionais, por possibilitar ganhos em competitividade e sustentabilidade do setor produtivo (Balbino et al., 2011b).

As vantagens desse sistema de cultivo de solo no Brasil são comprovadas pela viabilidade técnica, econômica, social e, principalmente, ambiental nos principais biomas, inclusive no Cerrado. Isso pode ser explicado pelas inúmeras combinações possíveis entre culturas agrícolas, criação animal e plantios florestais, conferindo grande aplicabilidade ao sistema e adaptabilidade à realidade de diferentes regiões (Balbino et al., 2012b).

Nesse sistema, a compreensão dos fatores que regulam a decomposição dos resíduos orgânicos depositados sobre o solo assume importante papel no manejo do solo. A temperatura e a precipitação são elementos climáticos de grande relevância para a deposição e decomposição da serapilheira e sua incorporação ao sistema solo-planta (Dick et al., 2015; Santos et al., 2017). A qualidade da serapilheira pode ser influenciada pelo tipo de cobertura florestal, manejo, concentrações de nutrientes e composição estrutural das espécies (Souza et al., 2016; Villa et al., 2016). Dessa forma, é possível elaborar técnicas de cultivo que melhorem a utilização de nutrientes contidos nos resíduos vegetais que formam a serapilheira (Laclau et al., 2013; Skorupa et al., 2015; Villa et al., 2016).

$\mathrm{O}$ uso de ILPF em áreas agrícolas, com o manejo de fertilidade adequado, favorece a formação ou recuperação das pastagens, a produção de grãos e o comércio de espécies florestais, como o eucalipto (Balbino et al., 2011b; 2012a). As árvores, ao longo do seu crescimento e desenvolvimento, desempenham um papel importante, que é a produção e a deposição de resíduos orgânicos, o que melhora a capacidade física, química e biológica do solo e contribui para a ciclagem de nutrientes (Cunha-Neto et al., 2013; Schumacher et al., 2013; Witschoreck et al., 2015). Além disso, para o componente pecuário, esse sistema acomoda microclima favorável, elevando o conforto térmico para os animais à sombra das árvores (Silva et al., 2011).

A quantidade de serapilheira e seu conteúdo de nutrientes incorporados ao solo dependem da capacidade produtiva do solo, das condições climáticas, do material genético e da idade das árvores e do espaçamento, entre outros fatores (Versini et al., 2013; Gatto et al., 2014; Ribeiro et al., 2018; Valadão et al., 2019). Dessa forma, os objetivos desse estudo foram avaliar a produção, decomposição e macronutrientes ( $\mathrm{N}, \mathrm{P}, \mathrm{K}, \mathrm{S}, \mathrm{Ca}$ e $\mathrm{Mg}$ ) da serapilheira entre árvores e entre renques em área de ILPF.

\section{Material e métodos}

O trabalho foi conduzido por 11 meses, no período de dezembro de 2011 a outubro de 2012, na área 
experimental de integração lavoura-pecuária-floresta (ILPF), localizada no Centro de Pesquisa Agropecuária da Embrapa Cerrados, em Planaltina, DF(15'36’38,82" S e $47^{\circ} 42^{\prime} 13,63^{\prime}$ 'W), como pode ser observado na Figura 1.

O solo da área de estudo é classificado como Latossolo Vermelho Distrófico (Santos et al., 2018). O clima da região, segundo a classificação de Köppen, é Aw, com estação seca bem definida (entre maio e setembro) e chuvosa (entre outubro e abril) (Alvares, 2014). A precipitação média anual é de $1.254 \mathrm{~mm}$, concentrada nos meses de outubro a abril, a temperatura média de $21,7^{\circ} \mathrm{C}$ e a velocidade do vento de $2,09 \mathrm{~m} \mathrm{~s}^{-1}$ (Figura 2), dados obtidos pela Estação Meteorológica da Embrapa Cerrados.

Cada unidade experimental possui, aproximadamente 1,4 ha com plantio de híbrido Eucalyptus urograndis, com dois anos e oito meses. A média do diâmetro a $1,30 \mathrm{~m}$ do solo (DAP) das árvores foi de $10 \mathrm{~cm} \mathrm{e}$
$13,6 \mathrm{~cm}$, e a altura média de 12,4 e $17,81 \mathrm{~m}$ para os anos de 2011 e 2012, respectivamente. Na posição entre renques foi cultivado sorgo em 2009 e soja em 2010, 2011 e 2012.

As coletas de campo foram realizadas no experimento de ILPF no tratamento com linhas duplas de plantio de Eucalyptus urograndis, com $12 \mathrm{~m}$ de distância entre as linhas entre árvores (Figura 3). Foram instalados coletores de serapilheira de formato quadrado de $50 \mathrm{~cm} \mathrm{x}$ $50 \mathrm{~cm}\left(0,25 \mathrm{~m}^{2}\right)$, confeccionados de madeira e sombrite. Para determinar a decomposição de serapilheira, foram instaladas 60 sacolas de decomposição (litterbags) e confeccionadas com malha de náilon $2 \mathrm{~mm}(20 \mathrm{~cm} \mathrm{x}$ $30 \mathrm{~cm}$ ). Essas foram posicionadas entre árvores e entre renques, dispostas no sentido leste e oeste do plantio das árvores de eucalipto. Foram coletadas quatro por mês, ao longo de 11 meses, e a serapilheira depositada foi coletada mensalmente, no mesmo período.

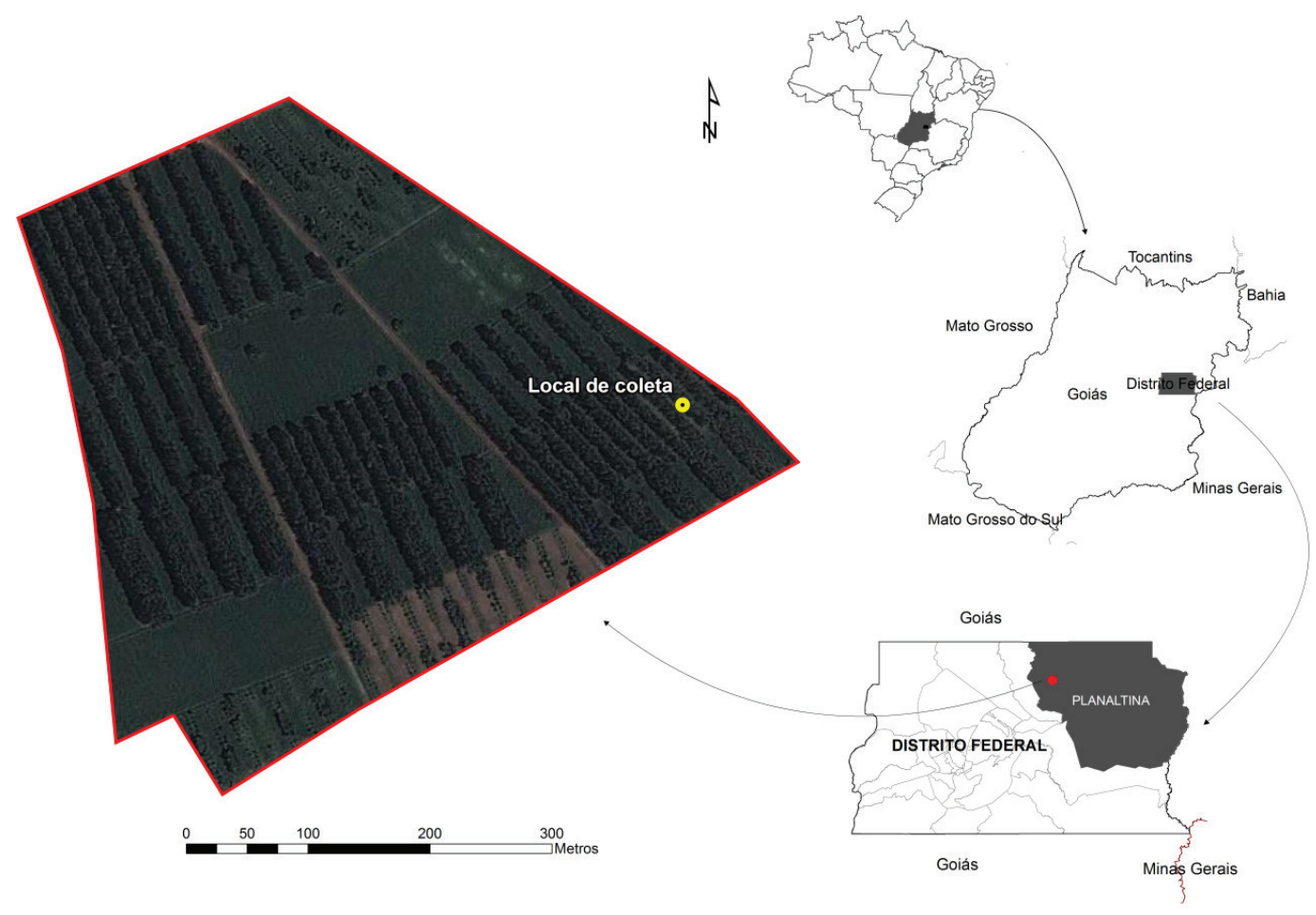

Figura 1. Localização da área experimental de integração lavoura-pecuária-floresta (ILPF), Embrapa Cerrados, Planaltina, Distrito Federal, Brasil. Fonte: IBGE (2007).

Figure 1. Location of the experimental area of crop-livestock-forestry integrated system (CLFI), Embrapa Cerrados, Planaltina, Federal Discrict, Brazil. Source: IBGE (2007). 


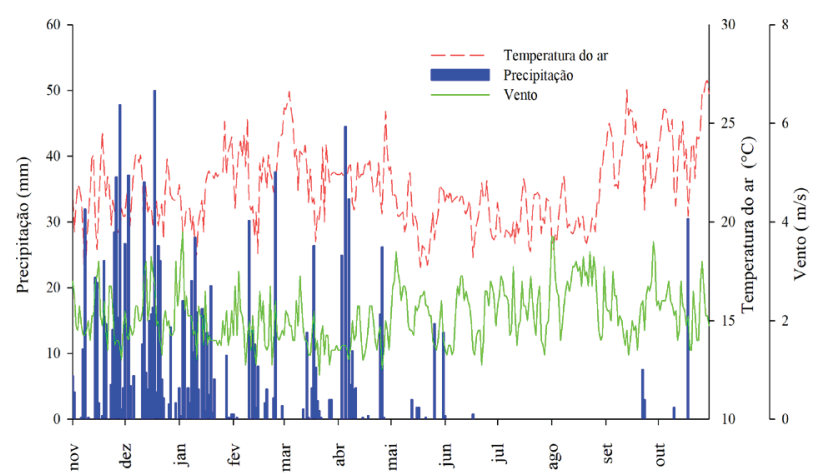

Figura 2. Precipitação, temperatura do ar e vento, Embrapa Cerrados, Planaltina, DF.

Figure 2. Precipitation, air temperature and wind, Embrapa Cerrados, Planaltina, Federal Discrict, Brazil.

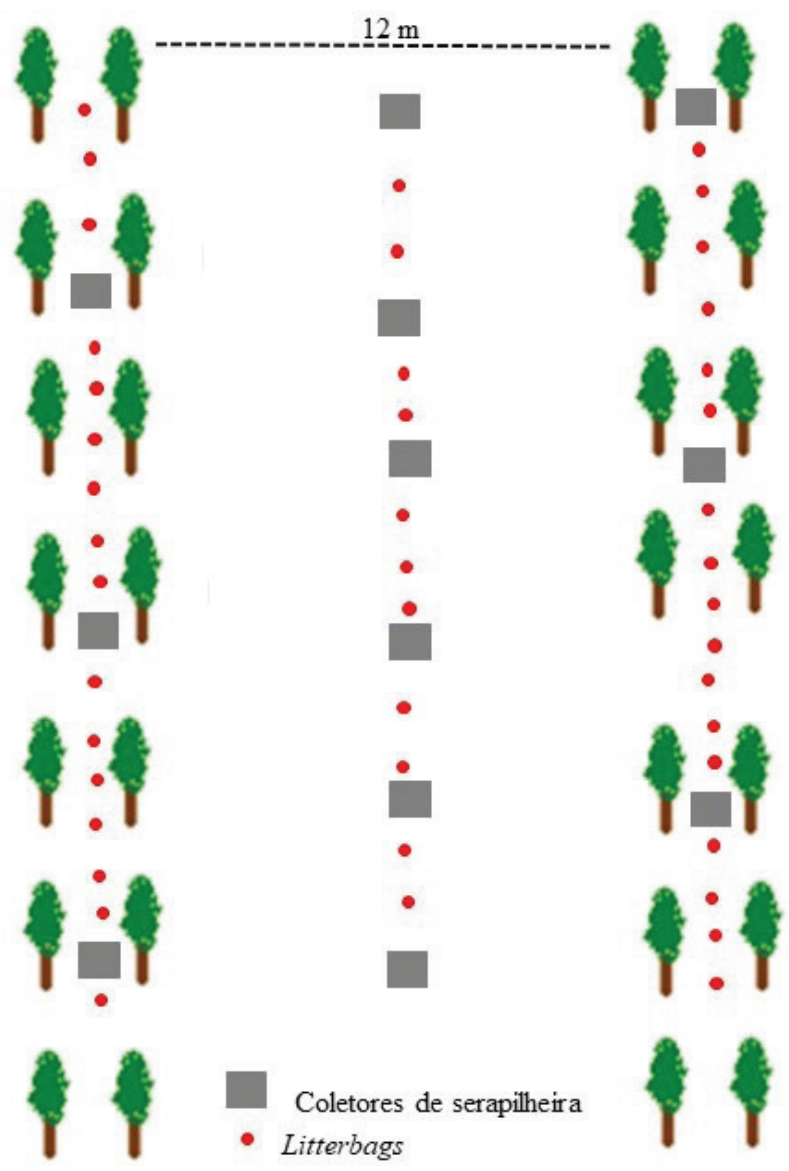

Figura 3. Localização dos coletores e litterbags de serapilheira no experimento de integração lavoura-pecuária-floresta (ILPF).

Figure 3. Litter traps and litterbags location in the croplivestock-forestry integrated system (CLFI) experiment.
A serapilheira, coletada foi acondicionada em sacos de papel e seca em estufa de circulação forçada de ar a $65^{\circ} \mathrm{C}$, até atingir peso constante. Posteriormente, o peso

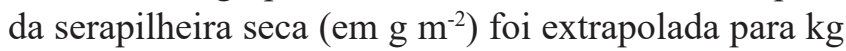
$\mathrm{ha}^{-1}$. A última coleta de serapilheira depositada e dos litterbags seria realizada em novembro de 2012, para completar um ano, mas não ocorreu devido ao incêndio ocorrido na área de estudo.

Em cada litterbag foram adicionados $20 \mathrm{~g}$ de material de serapilheira de Eucalyptus urograndis. Mensalmente, durante o período de dezembro de 2011 a outubro de 2012, coletou-se a serapilheira encontrada nos litterbags, que foi lavada e acondicionada em sacos de papel e seca em estufa a $65^{\circ} \mathrm{C}$, até atingir peso constante.

Os dados de decomposição e deposição de serapilheira de Eucalyptus urograndis foram submetidos à análise de variância pelo teste de F (ANOVA). As médias entre as posições (árvore e renques) foram comparadas pelo teste de Tukey $(\mathrm{p}<0,05)$.

Para calcular a taxa de decomposição (k), foi utilizado o modelo exponencial da soma de dois parâmetros (equação 1), utilizado por Plante \& Parton (2007):

$$
\mathrm{y}=\mathrm{A} \text { e-kAt }+\mathrm{B} \text { e-kBt }
$$

Em que y é a quantidade de matéria seca remanescente em ( $\left.\mathrm{kg} \mathrm{ha}^{-1}\right)$ após um período de tempo $\mathrm{t}$ (em dias); A e $\mathrm{B}$ são as constantes, $\mathrm{k}_{\mathrm{A}}$ é a constante de decomposição em um curto período e $\mathrm{k}_{\mathrm{B}}$ é a constante de decomposição recalcitrante, ambas obtidas pelo software Sigma Plot for Windows 12.5 através dos dados de serapilheira contidos nos litterbags ao longo de 345 dias.

Outra característica útil na avaliação da decomposição de materiais vegetais é o tempo de meia-vida, que expressa o período de tempo necessário para que metade dos resíduos se decomponha ou para que metade dos nutrientes contidos nesses resíduos seja liberada. De acordo com Olson (1983), é possível calcular os tempos de meia-vida pela equação:

$$
\mathrm{t}_{1 / 2}=\ln (2) / \mathrm{k}=0,693 / \mathrm{k}
$$

Em que $t_{1 / 2}$ é tempo de meia-vida de matéria remanescente; ln (2) é um valor constante; e k é a constante de decomposição. As equações matemáticas que melhor representam a decomposição de matéria seca foram obtidas por meio do software Sigma Plot. 
As amostras de serapilheira de deposição dos coletores de serapilheira e dos litterbags, foram moídas em moinho de navalha. Foram encaminhados cerca de $5 \mathrm{~g}$ do material para laboratório, onde foram realizadas as análises de macronutrientes $(\mathrm{N}, \mathrm{P}, \mathrm{K}, \mathrm{S}$, $\mathrm{Ca}$ e $\mathrm{Mg}$ ). $\mathrm{O} \mathrm{N}$ foi obtido pelo método de Kjeldahl, e os demais nutrientes foram submetidos pelos métodos de determinação definidos em Donagema et al. (2011). Os valores dos nutrientes encontrados na deposição e na decomposição foram submetidos à análise de variância, sendo as médias comparadas pelo teste de Tukey $(\mathrm{p}<0,05)$ com auxílio do programa Assistat 7.7.

Os dados de biomassa da serapilheira (depositada e decomposta) e os dados climatológicos da região, disponibilizados pela estação meteorológica da Embrapa Cerrados (temperatura média, vento e precipitação), foram submetidos à análise estatística de correlação de Pearson, em busca de associações entre essas variáveis.

\section{Resultados}

\section{Deposição de serapilheira}

A deposição mensal de serapilheira foi maior em agosto $\left(1.392 \mathrm{~kg} \mathrm{ha}^{-1}\right)$, referente aos coletores entre as árvores, e em junho ( $\left.867 \mathrm{~kg} \mathrm{ha}^{-1}\right)$, entre renques. Os maiores valores de deposição de serapilheira ocorreram com os menores índices pluviométricos, a partir de junho de 2012 (Figura 4).

Entre novembro de 2011 e setembro de 2012 obtevese um total de serapilheira acumulada de $9.225,2 \mathrm{~kg} \mathrm{ha}^{-1}$ entre árvores e $4.137,8 \mathrm{~kg} \mathrm{ha}^{-1}$ entre renques, com registro de precipitação média anual de $1.471,5 \mathrm{~mm}$ no período. A média de deposição da serapilheira para as posições entre árvores e entre renques no sistema de integração lavoura-pecuária-floresta (ILPF) foi de $6.681,5 \mathrm{~kg} \mathrm{ha}^{-1} \mathrm{em} 11$ meses de avaliação, com maiores deposições entre árvores, exceto em novembro e dezembro de 2011.

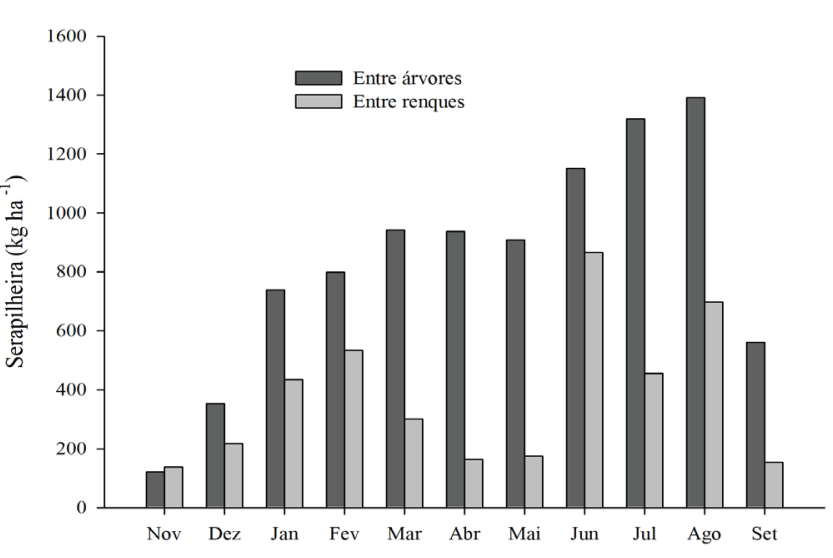

Figura 4. Deposição mensal de serapilheira na área de integração lavoura-pecuária-floresta, na Embrapa Cerrados, Planaltina, DF.

Figure 4. Monthly litterfall in the crop-livestock-forest integration area at Embrapa Cerrados, Planaltina, Federal Discrict, Brazil.

\section{Decomposição de serapilheira}

O modelo matemático exponencial, que descreve a velocidade de decomposição entre árvores e entre renques em plantio de Eucalyptus urograndis na área experimental de ILPF está apresentado na Figura 5. A constante de decomposição de curto período $\left(\mathrm{k}_{\mathrm{A}}\right)$ e a constante de decomposição mais recalcitrante $\left(\mathrm{k}_{\mathrm{B}}\right)$ foram, respectivamente, de 0,0701 e 0,0005 entre árvores e de 0,0687 e 0,0006 entre renques. O tempo de meia vida da decomposição $\left(\mathrm{t}_{1 / 2}\right) \mathrm{k}_{\mathrm{A}}$ e $\mathrm{k}_{\mathrm{B}}$ foram, respectivamente, de 10 e 1.386 dias entre árvores e de 10 e 1.155 dias entre renques.

Adecomposição da serapilheira após 345 dias apresentou $66,2 \%$ e $62,9 \%$ de serapilheira remanescente para as posições entreárvores (EA) e entre renques (ER), respectivamente. A maior transformação de serapilheira, após a instalação dos litterbags, ocorreu nos primeiros 30 dias (Figura 5). 


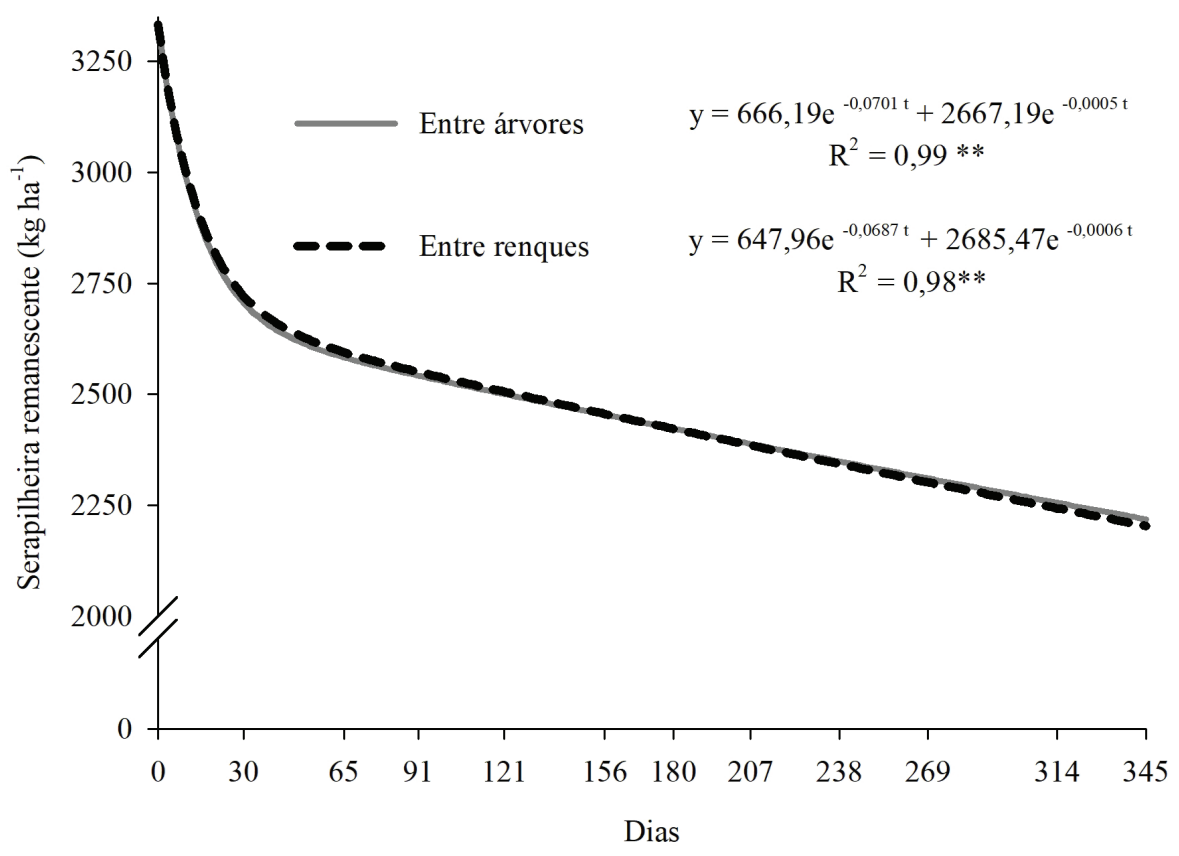

Figura 5. Curva de decomposição de serapilheira entre árvores e entre renques na área experimental de integração lavourapecuária-floresta, na Embrapa Cerrados, Planaltina, DF. ** = significativo a 1\% de probabilidade.

Figure 5. Litter decomposition curve between trees and between rows in the experimental area of crop-livestock-forest integration area at Embrapa Cerrados, Planaltina, Federal Discrict, Brazil. ** = significant at 1\% of probability.

Foi observada correlação da serapilheira (produção e decomposição) entre árvores e entre renques apenas com precipitação, dentre as variáveis climatológicas testadas (temperatura, vento e precipitação), como pode ser observado na Tabela 2. Verificou-se correlação negativa entre a precipitação e a produção de serapilheira entre árvores e correlações positivas entre árvores e entre renques para decomposição.

Tabela 2. Matriz de correlação de Pearson entre as variáveis climatológicas em relação à serapilheira (deposição e decomposição) na área experimental de integração lavoura-pecuária-floresta, na Embrapa Cerrados, Planaltina, DF.

Table 2. Pearson correlation matrix between climatic variables in relation to litter (litterfall and decomposition) in the experimental area of crop-livestock-forest integration area at Embrapa Cerrados, Planaltina, Federal Discrict, Brazil.

\begin{tabular}{|c|c|c|c|c|c|c|c|}
\hline \multirow{2}{*}{ Elementos } & \multirow{2}{*}{ Temperatura } & \multirow{2}{*}{ Vento } & \multirow{2}{*}{ Precipitação } & \multicolumn{4}{|c|}{ Serapilheira } \\
\hline & & & & $\mathbf{E A}^{1}$ & $\mathbf{E R}^{2}$ & $\mathbf{E A}^{1}$ & $\mathbf{E R}^{2}$ \\
\hline & & & & \multicolumn{2}{|c|}{ deposição } & \multicolumn{2}{|c|}{ decomposição } \\
\hline Temperatura & 1 & $0,35^{\mathrm{ns}}$ & $0,49^{\text {ns }}$ & $-0,11^{\mathrm{ns}}$ & $0,11^{\text {ns }}$ & $-0,37^{\mathrm{ns}}$ & $-0,37^{\text {ns }}$ \\
\hline Vento & & 1 & $0,31^{\mathrm{ns}}$ & 0,29 ns & 0,07 ns & $-0,27^{\mathrm{ns}}$ & $-0,38^{\mathrm{ns}}$ \\
\hline Precipitação & & & 1 & $-0,79 * *$ & $0,44^{\text {ns }}$ & $0,80^{* *}$ & $0,72 *$ \\
\hline EA & & & & 1 & & & \\
\hline ER & & & & & 1 & & \\
\hline
\end{tabular}

$\mathrm{EA}=$ entre árvores; $\mathrm{ER}=$ entre renques; $\mathrm{ns}=$ não significativo, ${ }^{*} \mathrm{e} * *$ significativo a $5 \% \mathrm{e} 1 \%$, respectivamente, pelo teste $\mathrm{t}$. 
Nutrientes na deposição e decomposição de serapilheira

$\mathrm{Na}$ deposição de serapilheira, o conteúdo de todos os macronutrientes ( $\mathrm{N}, \mathrm{P}, \mathrm{K}, \mathrm{S}, \mathrm{Ca}$ e $\mathrm{Mg}$ ) foi maior na posição entre as árvores. Após 11 meses de decomposição, os conteúdos de macronutrientes não diferiram estatisticamente quanto às posições avaliadas. $\mathrm{O}$ Ca foi o elemento que apresentou maior conteúdo na avaliação de deposição e decomposição de serapilheira, independentemente da posição. Os níveis de P foram os mais baixos na deposição e decomposição (Tabela 3).

Tabela 3. Médias de conteúdo anual de macronutrientes na deposição e decomposição de serapilheira, na área experimental de integração lavoura-pecuária-floresta, na Embrapa Cerrados, Planaltina, DF.

Table 3. Annual macronutrient content averages in litterfall and decomposition in the experimental area of crop-livestockforest Integration area at Embrapa Cerrados, Planaltina, Federal Discrict, Brazil.

\begin{tabular}{|c|c|c|c|c|c|c|}
\hline \multirow{2}{*}{ Posição } & $\mathbf{N}$ & $\mathbf{P}$ & $\mathbf{K}$ & $\mathbf{C a}$ & Mg & $\mathbf{S}$ \\
\hline & -- & & $----k g$ & $a^{-1}-$ & & \\
\hline & \multicolumn{6}{|c|}{ Deposição da serapilheira* } \\
\hline $\mathrm{EA}^{1}$ & 90,70 a & $6,47 \mathrm{a}$ & 44,42 a & $116,62 \mathrm{a}$ & $15,73 \mathrm{a}$ & $\begin{array}{c}10,18 \\
\mathrm{a}\end{array}$ \\
\hline $\mathrm{ER}^{2}$ & $46,01 \mathrm{~b}$ & $3,39 \mathrm{~b}$ & $24,70 \mathrm{~b}$ & $54,78 \mathrm{~b}$ & $8,23 \mathrm{~b}$ & $5,32 \mathrm{~b}$ \\
\hline \multirow[t]{2}{*}{ CV $(\%)$} & 16,04 & 15,95 & 16,14 & 15,92 & 15,82 & 15,96 \\
\hline & \multicolumn{6}{|c|}{ Decomposição da serapilheira* } \\
\hline $\mathrm{EA}^{1}$ & 24,89 a & $1,14 \mathrm{a}$ & $2,23 \mathrm{a}$ & 32,96 a & $3,02 \mathrm{a}$ & $3,53 \mathrm{a}$ \\
\hline $\mathrm{ER}^{2}$ & 26,24 a & $0,93 \mathrm{a}$ & $1,95 \mathrm{a}$ & 34,87 a & $2,77 \mathrm{a}$ & $4,16 \mathrm{a}$ \\
\hline CV $(\%)$ & 10,14 & 26,48 & 21,71 & 10,25 & $-8,68$ & $-21,56$ \\
\hline
\end{tabular}

$\mathrm{EA}=$ entre árvores; $=\mathrm{ER}=$ entre renques; $\mathrm{CV}=$ coeficiente de variação. Médias seguidas pela mesma letra na coluna não diferem estatisticamente pelo teste de Tukey $(\mathrm{p}<0,05){ }^{*}=$ número de amostras analisadas para determinação dos nutrientes $(=11)$.

\section{Discussão}

O componente arbóreo (Eucalyptus urograndis) contribuiu para melhorar a disponibilidade de nutrientes via serapilheira em sistema de integração lavourapecuária-floresta (ILPF), pois foram registrados maiores deposição e conteúdo de nutrientes na serapilheira na posição entre árvores (Figura 4 e Tabela 3). Segundo Valadão et al. (2021), a deposição de serapilheira produzida por plantios de eucalipto contribui para o aporte de matéria orgânica sob em solos do Cerrado. A influência do componente arbóreo e a distância da linha de plantio das árvores dos sistemas agrossilvipastoris na deposição de serapilheira e de nutrientes no solo também foi relatada em estudo desenvolvido por Freitas et al. (2013).

O espaçamento, dentre outros fatores, afetou a quantidade e qualidade de serapilheira que caem da parte aérea de Eucalyptus urograndis (Figura 4 e Tabela 2). Os espaçamentos estão diretamente relacionados à quantidade de serapilheira produzida em plantios de eucaliptos (Silveira et al., 2014). Freitas et al. (2013) também encontraram maior deposição de serapilheira em espaçamentos menores em sistema agrossilvipastoril com eucalipto. Portanto, a escolha do arranjo e espaçamento das linhas de plantio das árvores em sistemas consorciados pode beneficiar a ciclagem de nutrientes advindos da serapilheira.

O clima também pode ser um regulador importante da deposição e decomposição de serapilheira. A maior deposição ocorreu na estação seca, resultante da redução da precipitação (Figura 2), que acarreta ao ecossistema florestal um estresse hídrico e a consequencia queda de folhas para diminuir seu consumo de água (Giácomo et al., 2012). As variáveis climatológicas, em especial a precipitação, têm estreita relação com a deposição e decomposição serapilheira. Em uma escala mais ampla, a biomassa vegetal é determinada pela disponibilidade de água no solo, que é influenciada pela distribuição das chuvas. Em estudo realizado em área de eucalipto na região do Cerrado, Ribeiro et al. (2018) também verificaram alta correlação entre precipitação e quantidade de serapilheira depositada.

Não foram observadas diferenças na decomposição de serapilheira entre as posições estudadas. Possivelmente, esse resultado se deve ao curto período de avalição. Segundo Ribeiro et al. (2018), estudos de decomposição de espécies florestais sob as condições ambientais da região do Cerrado, necessitam de pelo menos dois anos de avaliação.

De maneira geral, a decomposição da serapilheira em ILPF também depende das condições climáticas. Em condições de boa disponibilidade de água, durante a época chuvosa, há o favorecimento da decomposição. A decomposição de serapilheira, observada em nossa área de estudo, segue um padrão similar a monocultivos de eucaliptos no Cerrado. As pesquisas mostram baixa taxa de decomposição de serapilheira, com aumentos das taxas registrados imediatamente após as primeiras 
chuvas (Souza et al., 2016; Ribeiro et al., 2018; Valadão et al., 2019).

A maior decomposição de serapilheira registrada nos primeiros meses pode estar associada à alta atividade dos agentes decompositores da macrofauna, mesofauna e microrganismos do solo (Swift et al., 2010; Korasaki et al., 2013). Após esse período, grande parte das estruturas mais recalcitrantes, ricas em lignina, celulose, assim como nervuras e pecíolos, ainda permanecem, diminuindo a velocidade de decomposição (Viera et al., 2014; Lima et al., 2015).

$\mathrm{Na}$ posição entre árvores, o conteúdo de $\mathrm{N}$ foi superior para deposição de serapilheira (Tabela 2). Esses resultados corroboram com os de Freitas et al. (2013), que encontraram maior teor de $\mathrm{N}$ em serapilheira no sistema agrossilvipastoril com eucalipto, em comparação com o sistema que possui apenas eucalipto.

Apesar do $\mathrm{N}$ ser um elemento extremamente móvel na planta, é um dos elementos que apresentou maior retorno ao solo (Tabela 2), que pode ser decorrente da grande dinâmica que esse elemento possui no sistema, da adubação que ocorreu na área, ou até mesmo devido ao teor de matéria orgânica do solo. $\mathrm{O} \mathrm{N}$ é um dos elementos que apresenta maior teor nas células foliares e mesmo sendo reciclado, boa parte ainda permanece nos tecidos senescentes das plantas (Cantarella, 2007). Com o elevado custo de fertilizantes, áreas de ILPF com espécies fixadoras de $\mathrm{N}$ surgem como opção para melhorar a fertilidade do solo e reduzir a necessidade de fertilizantes (Balbino et al., 2012a). Os nutrientes P, Ca, $\mathrm{K}, \mathrm{Mg}$ e $\mathrm{S}$ também foram superiores na posição entre árvores. Segundo Freitas et al (2016), os estoques de nutrientes em sistemas de plantio florestal correspondem aos encontrados em vegetação nativa.

\section{Conclusões}

A maior deposição de serapilheira e adição de nutrientes ocorreram na posição entre árvores. Assim, a introdução do componente arbóreo em sistemas agropecuários pode auxiliar na melhoria das propriedades físico/químicas do solo, destacando a ciclagem e o aporte de nutrientes. Estes propiciam aumento da produção agrícola e da qualidade nutricional da pastagem, refletindo na capacidade de suporte animal no sistema de integração lavoura-pecuária-floresta.

\section{Agradecimentos}

Embrapa Cerrados e Programa de Pós Graduação de Ciências Florestais da Universidade de Brasília (UnB).

\section{Referências}

Alvares, C. A. et al. Köppen's climateclassification map for Brazil. Meteorologische Zeitschrift, v. 22, p. 711-728, 2014. https://doi. org/10.1127/0941-2948/2013/0507.

Balbino, L. C. et al. Agricultura sustentável por meio da Integração Lavoura-Pecuária-Floresta (ILPF). Informações Agronômicas IPNI, n. 138, p. 1-18, 2012a.

Balbino, L. C. et al. Evolução tecnológica e arranjos produtivos de sistemas de integração lavoura-pecuária-floresta no Brasil. Pesquisa Agropecuária Brasileira, v. 46, n. 10, p. 1-12, 2011a. https://doi. org/10.1590/S0100-204X2011001000001.

Balbino, L. C. et al. Marco referencial: integração lavoura-pecuáriafloresta (iLPF). Brasília, DF: Embrapa, 2011b. 130 p.

Balbino, L. C. et al. Sistemas de integração: o que são, suas vantagens e limitações. In: Bungenstab, D. Sistemas de integração a produção sustentável: integração lavoura-pecuária-floresta (iLPF). 2 ed. Brasília, DF: Embrapa, 2012b. p. 11-18.

Cantarella, H. Nitrogênio. In: Novais, R. F. et al. Fertilidade do solo. Viçosa, MG: Sociedade Brasileira de Ciência do Solo, 2007. p. $375-470$.

Colodette, J. L. et al. The Brazilian wood biomass supply and utilization focusing on eucalypt. Chemical and Biological Technologies in Agriculture, v. 25, v. 1, p. 1-8, 2014. https://doi. org/10.1186/s40538-014-0025-x.

Cunha-Neto, F. V. et al. Acúmulo e decomposição da serapilheira em quatro formações florestais. Ciência Florestal, v. 23, n. 3, p. 379-387, 2013. http://dx.doi.org/10.5902/1980509810549.

Dick, G. et al. Litterfall of a Subtropical Seasonal Forest in northern Rio Grande do Sul. Ecologia e Nutrição Florestal, v. 3, n. 1, p. $1-8,2015$.

Donagema, G. K. et al. (org.). Manual de métodos de análise de solo. 2. ed. rev. Rio de Janeiro: Embrapa Solos, 2011. 230 p. (Embrapa Solos. Documentos, 132).

FAO. Global forest resources assessment FRA 2015: how are the world's forests changing? Rome, 2015. 46 p. Disponível em: http:// www.fao.org/3/a-i4793e.pdf. Acesso em: 25 jan. 2018.

Ferreira, G. W. D. et al. Nutrient release from decomposing Eucalyptus harvest residues following simulated management practices in multiple sites in Brazil. Forest Ecology and Management, v. 370, n. 15, p. 1-11. 2016. https://doi.org/10.1016/j.foreco.2016.03.047.

Freitas, E. C. S. et al. Deposição de serapilheira e de nutrientes no solo em Sistema Agrossilvipastoril com eucalipto e acácia. Revista Árvore, v. 37, n. 3, p. 409-417, 2013. http://dx.doi.org/10.1590/ S0100-67622013000300004. 
Deposição, decomposição e conteúdo de nutrientes de serapilheira em área de integração lavoura-pecuária-floresta na região do 9 de 9 Cerrado

Freitas, I. C. de. Carbono no solo, acúmulo e qualidade da serapilheira em sistemas de produção familiar. Floresta, v. 46, n. 1, p. 31-38, 2016. http://dx.doi.org/10.5380/rf.v46i1.42065.

Gatto, A. et al. Ciclagem e balanço de nutrientes no sistema solo-planta em um plantio de Eucalyptus sp. no Distrito Federal. Revista Brasileira de Ciência do Solo, v. 38, n. 3, p. 879-887, 2014. http://dx.doi.org/10.1590/ S0100-06832014000300019.

Giácomo, R. G. et al. Aporte e decomposição de serapilheira em áreas de Cerradão e Mata Mesofítica na Estação Ecológica de PirapitingaMG. Ciência Florestal, v. 22, n. 4, p. 669-680, 2012. http://dx.doi. org/10.5902/198050987549.

Gonçalves, J. L. M. et al. Integrating genetic and silvicultural strategies to minimize abiotic and biotic constraints in Brazilian eucalypt plantations. Forest Ecology and Management, v. 301, n. 1, p. 6-27, 2013. https:// doi.org/10.1016/j.foreco.2012.12.030.

IBÁ. Industria Brasileira de árvores. Relatório 2019. Disponível em: https:/iba.org/datafiles/publicacoes/relatorios/iba-relatorioanual2019. pdf. Acesso em: 25 mar. 2019.

Korasaki, V. et al. Macrofauna. In: Moreira, F. M. S. et al. (ed.). O ecossistema solo: componentes, relações ecológicas e efeitos na produção vegetal. Lavras: Ed. da UFLA, 2013. p. 79-128.

Laclau, J. et al. Mixing Eucalyptus and Acacia trees leads to fine root over-yielding and vertical segregation between species. Oecologia, v. 172, n. 3, p. 903-913, 2013. https://doi.org/10.1007/s00442-012-2526-2.

Lima, R. P. et al. Aporte e decomposição da serapilheira na Caatinga no Sul do Piauí. Floresta e Ambiente, v. 22, p. 42-49, 2015. http://dx.doi. org/10.1590/2179-8087.062013.

Martins, E. C. A. et al. Alterações dos atributos físico-químicos da camada superficial do solo em resposta à agricultura com soja na Várzea do Tocantins. Biota Amazônia, v. 5, n. 4. p. 56-62, 2015.

Olson J. S. Energy storage and the balance of producers and decomposers in ecological systems. Ecology, v. 44, p. 322-331, 1963. https://doi. org/10.2307/1932179.

Ouyang, Y. et al. A system dynamic model to estimate hydrological processes and water use in a eucalypt plantation. Ecological Engineering, v. 86, p. 290-299, 2016. https://doi.org/10.1016/j.ecoleng.2015.11.008.

Plante, A. F. \& Parton, W. J. The dynamics of soil organic matter and nutriente cycling. In: Paul, E.A. Soil Microbiology, Ecology, and Biochemistry. 3 ed. Burlington: Academic Press, 2007. p. 433-467.

Ribeiro, F. P. et al. Litter dynamics in Eucalyptus and native forest in the Brazilian Cerrado. Journal of Agricultural Science, v. 10, p. 1-15, 2018. https://doi.org/10.5539/jas.v10n11p29.

Santos, A. F. A. et al. Eucalyptus litter capacity of stock and water retention.. Floresta e Ambiente, v. 24, p. 1-9, 2017. http://dx.doi. org/10.1590/2179-8087.030315.
Santos, G. A. et al. Adaptabilidade de híbridos multiespécies de eucalyptus ao Estado do Rio Grande do Sul. Revista Árvore, v, 37, n. 4, p. 759-769, 2013. https://doi.org/10.1590/S0100-67622013000400019.

Santos, H. G. dos et al. Sistema Brasileiro de Classificação de Solos. 5. ed. Brasília, DF: Embrapa, 2018. 356 p.

Schumacher M. V. et al. Produção e decomposição de serapilheira em um povoamento de eucalyptus urophylla $\mathrm{x}$ encalyptus globulus maidenii. Revista Cerne, v. 19, p. 501-508, 2013. http://dx.doi.org/10.1590/S010477602013000300018.

Silva, J. A. R. et al. Conforto térmico de búfalas em sistema silvipastoril na Amazônia Oriental. Pesquisa Agropecuária Brasileira, v. 46, p. 1364-1371, 2011. https://doi.org/10.1590/S0100-204X2011001000033.

Silveira, E.R. et al. Efeito do espaçamento de plantio na produção de madeira e serapilheira de Eucalyptus dunni na região sudoeste do Paraná. Revista Técnico-Científica do CREA-PR, v. 2, p. 1-0, 2014.

Skorupa, A. L. A. et al. Decomposição de serapilheira florestal: efeito da idade do eucalipto e topografia no sudeste do Brasil. Revista Árvore, v. 39, p. 1055-1064, 2015. http://dx.doi.org/10.1590/010067622015000600008.

Souza, J. V. et al. Stock and litter decomposition in different vegetation types and eucalypt plantations in the cerrado region, Brazil. Australian Journal of Basic and Applied Sciences, v. 10, p. 74-81, 2016.

Swift, M. J. et al. Inventário da biodiversidade biológica do solo: conceitos e orientações gerais. In: Moreira, F. M. (ed.). Manual de biologia dos solos tropicais: amostragem e caracterização da biodiversidade. Lavras: Ed. da UFLA, 2010. p. 23-41.

Valadão, M. B. X. et al. Litterfall, litter layer and leaf decomposition in Eucalyptus stands on Cerrado soils. Scientia Forestalis, v. 46, n. 121, p. 256-264, 2019. DOI: doi.org/10.18671/scifor.v47n122.08.

Valadão, M. B. X. et al. Litter layer removal and implications on decomposition in a Eucalyptus stand in the Cerrado. Scientia Forestalis, v. 49, n. 131, p. 1-13, 2021. DOI: https://doi.org/10.18671/scifor. $\mathrm{v} 49 \mathrm{n} 131.08$

Versini, A. et al. The manipulation of organic residues affects tree growth and heterotrophic $\mathrm{CO}_{2}$ efflux in a tropical Eucalyptus plantation. Forest Ecology and Management, v. 301, p. 79-88, 2013. https://doi. org/10.1016/j.foreco.2012.07.045.

Viera M. et al. Disponibilização de nutrientes via decomposição da serapilheira foliar em um plantio de Eucalyptus urophylla $\mathrm{x}$ Eucalyptus globulus. Revista Floresta e Ambiente, v. 21, p. 307-315, 2014. http:// dx.doi.org/10.1590/2179-8087.066313.

Villa, E. B. et al. Aporte de serapilheira e nutrientes em área de restauração florestal com diferentes espaçamentos de plantio. Floresta Ambiente, v. 23, p. 90-99, 2016. http://dx.doi.org/10.1590/2179-8087.067513.

Witschoreck, R. \& Schumacher, M. V. Alocação de nutrientes em povoamentos de Eucalyptus saligna Sm. na região de Guaíba - Rio Grande do Sul. Cerne, v. 21, p. 625-63, 2015. http://dx.doi.org/10.1590 /01047760201521041963. 